\title{
Use of Kinect Tool to Measure the Physical Capabilities of Patients for Wolf Motor Function Test in Centre Hospitalier Universitaire de Grenoble (CHU)
}

\author{
Amirmahdi TAHERI ${ }^{* 1}$, Julien VEYTIZOU ${ }^{1}$, Guillaume THOMANN ${ }^{1}$, Antoine THURIOT ${ }^{2}$ \\ ${ }^{1}$ Laboratoire des sciences pour la conception, l'optimisation et la production (G-SCOP) \\ CNRS: UMR5272, Institut National Polytechnique de Grenoble (INPG), \\ Université Joseph Fourier - Grenoble I, France \\ ${ }^{2}$ Ecole de kinésithérapie du Centre Hospitalier Universitaire de Grenoble (CHU), France
}

http://dx.doi.org/10.15221/14.390

\begin{abstract}
This study was performed in the G-SCOP Research laboratory in collaboration with Grenoble University Hospital Center (CHU) in France. The objective is to develop a tool for the automatic evaluation of motor skills of patients in clinical settings. The proposed method takes place in the context of medical activities carried out daily at this Hospital. Our study was in physiotherapy department to standardize the assessment phases of motor skills using Microsoft Kinect sensor. In our research, physiotherapists proposed to work on the WMFT (The Wolf Motor Function Test) measurement protocol which is recognized and used regularly for patient evaluation. In the first stage, it is tried to understand the protocol together with required elements. Then, a Kinect tool is prepared for the model which can be able to connect two points: the combination of the Wolf Motor Function Test (WMFT) with the Kinect output. Our initial experimental results of testing on both patients and 10 healthy persons in the G-SCOP laboratory showed the capability of the tool to measure the activities of the WMFT protocol. Furthermore, the results of this study can be the bases of future researches for the use of the Kinect in this domain and implementation of this tool directly in the Grenoble hospital.
\end{abstract}

Keywords: Microsoft Kinect, Wolf Motor function Test (WMFT), Motion analysis, Rehabilitation, Motor skills

\section{Introduction}

Scientific advances and progresses in the domains of engineering and use of technology have many consequences in the medical activities. As an instance, it allows the development of several equipment and facilities to examine patients in medical domain. However such equipment is often expensive, difficult to transport and time consuming in both installation and scanning. Standard 3D camera systems [1] have advantages but they present potential problems such as the use of markers, including soft tissue artifact, setup time and potentially uncomfortable exposure of the body's areas. Moreover, the evaluation made by the practitioners is unrepeatable, often human dependent.

Nevertheless, regarding to a recent development in computer gaming technology, the Microsoft Kinect is an inexpensive and portable device which no require markers to determine anatomical landmarks [2]. The accuracy of Kinect in medical applications has been proved to be sufficient; Oliveira et al. [3] proved that Kinect depth maps are accurate enough to be used in medical diagnosing. Zolfagharnasab et al. [4] proposed a framework to reconstruct female torso by using depth maps. The conducted research so far includes: accuracy, advantages and limitations of using Kinect for measuring physical movements of a patient. Moreover, the work includes the validity of the Microsoft Kinect for assessment of postural control [5] and conclusions about different angles accuracy and precision measurement with two different ranges in front of the Kinect sensor [6].

The work we discuss takes place in the context of medical activities carried out daily at Grenoble Hospital in France. It is aimed to standardize the assessment phases of motor skills using the Kinect tool. In this research we focus our study on the physiotherapy applications where physiotherapists use some predefined protocol to analyze the body under a standard such as MFM, WMFT and so on. In our research, physiotherapists proposed to work on the WMFT (The Wolf Motor Function Test) which is used regularly for patient evaluation. It allows health professionals to assess motor skills of patients but also their developments and progress.

\footnotetext{
*E-mail: Amirmahdi.Taheri@grenoble-inp.org
} 
The medical specialists whom we work with in Grenoble Hospital have already validated Kinect-based Rehabilitation Training Assistant System Research and Implementation [7]; consequently it may overcome the limitations thanks to the G-SCOP based movement analysis systems. In this regard, during recent studies on Kinect and its use in the medical field, researchers from the G-SCOP laboratory have worked on Kinect-related tools to record patients' movements via four considered degrees on freedom as follows: positional and temporal axis.

Previous measurements were conducted by physiotherapists comprised simple visual evaluated activities; however 3D camera apparatuses were used in case of more complicated activities. A number of problems exist in using 3D cameras such as issues with the use of markers, tag of the required tools. Moreover, output data of these tools may not be useful in their raw format and might require further processing (such as modeling techniques), algorithms, and so on.

In this study, we used the capabilities of Kinect tool for analyzing different parts of body and their features such as installation time, Portability, ease of use, lower price comparing with other 3D cameras. And also following recent researches in G-SCOP laboratory on the Kinect, we propose to use Kinect tool to measure the physical capabilities of patients for the protocol WMFT.

Our prospective for the research is how we can determine the activities of WMFT by using Kinect. The second section of this article is a review of literature. In the third stage we focus on the modeling by Kinect. According to the definition of the WMFT and Kinect tools, in this part we chose one WMFT test. For each score we defined the important joint according to the simple example which has been done by the use of Kinect and also discussed with physiotherapist. Finally, initial results are presented and followed by a conclusion.

\section{Literature Review}

In recent years, lot of studies has been performed to introduce a system to measure disability using Kinect. In 2012, Dutta et al. [8] have evaluated Kinect for 3-D kinematic measurement in the workplace and further Su et al. [9] proposed a framework with Kinect to compared two movements with each other. In these studies, researchers' activities are made in front of the Kinect, not using any element of standard practice and protocol.

In our case, we have two different elements: WMFT protocol and Kinect tool. For this reason, we propose two parts in this section: a combination of the WMFT with the output of the Kinect which is divided into medicine activities tests and engineering tools.

\subsection{Medical activities}

The recovery of motor function is thus a pivotal factor in rehabilitation of post-stroke patients. The WMFT has been introduced to satisfy such aim. This protocol has been developed in 1989 by Wolf et al, [10]. This test was designed to assess the motor ability of patients with moderate to severe upper extremity motor deficits in the laboratory and clinic.

Proposing in this research, a new version of the WFMT Protocol consists of 15 tasks with increasing complexity and two force tests [11]. For each task, the patients have to do some exercises with different parts of their body. Depending on the level of the activities, the practitioner has to evaluate the patient in a 5 level score, as depicted in figure 1, from the score 1 to 5 .

In case of misinterpretation of an activity, it should be repeated again. To cover delays, additional 120 seconds are considered as compensation. 
Score 0: Inability to do the test with upper limb tested.

Score 1: Upper limb tested does not participate functionally, but tries to use his upper limb.

Score 2: The patient is capable of performing the test, but with the assistance of untested for minor adjustments or a change in positioning of arms, or need more than 2 trials to complete the test, or to carry out very slowly.

Score 3: The patient is capable to performing the test, but the movement is influenced by a degree of synergy or is performed slowly and / or effort.

Score 4: The patient is capable of performing the test. The movement is slow but subnormal with less precision, coordination or fluidity.

Score 5: The patient is capable of performing the test, the movement seems normal.

Figure 1: Capacity scale.

\subsection{Engineering tools}

The version of Kinect which we used was launched on November 2010 by Microsoft. Based on a webcam style add-on peripheral for the Xbox 360 console, it enables users to interact and control with the tool without using a physical controller through a natural user interface using gestures and voice commands.

The Kinect sensor provides a genuinely new way to make sense of the world around it. The sensor recognizes the user's gestures and voice commands. The sensor recognizes the user's gestures using depth camera. Depth maps are captured from the reflection of IF projector mounted within the sensor [12].

Moreover, Kinect is able to find the skeletal tracking for transferring movements from a real person in the digital environment. The skeletal joint in the Kinect can monitor the person movements in real time. As we can see in figure 2, each point is called "joint" and the Kinect tool divides the body into 20 joints. The process was designed by G-SCOP's researchers, they were able to record individuals' movements via four considered degrees on freedom for each joints.

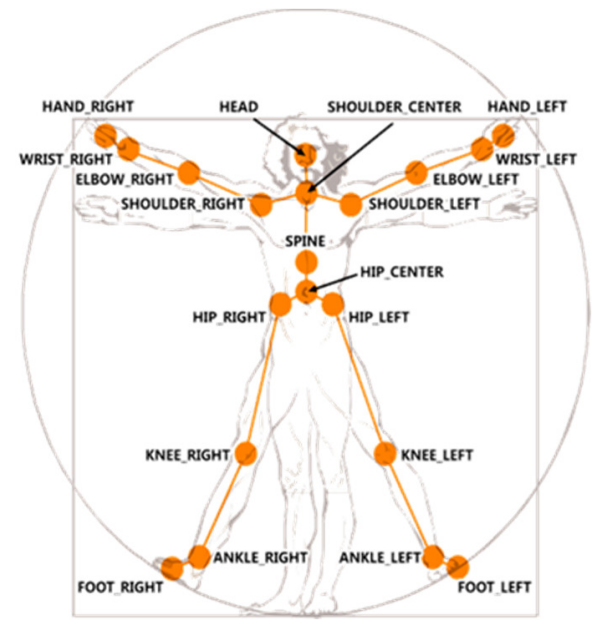

Figure 2: 20 joints. 


\section{Methodology}

As it is depicted Figure 3, the first experiments that have been done with the Kinect allows medical specialists to validate the proposed technology. The implementation of the Microsoft Kinect tool in the hospital has been developed with the respect of two main constraints:

1- The use of the Kinect outputs (for exploitation and capitalization) and

2- The use of the standard of WMFT protocol.

That is why the research methodology proposed is composed of two parts: a combination of the WMFT with Kinect output.

Currently, the researchers in G-SCOP laboratory were able to record movements of the patients in four variables which are time and $X, Y, Z$ axes positions for 20 points of body. The distance $Z$ is the distance between the Kinect tool and the patient, the $X-Y$ plan is perpendicular to the $Z$ axis, passed through the patient. As the first stage of the work, practitioners and researchers have chosen the fifth test of this WMFT protocol to verify the feasibility of the research. Thus, for each score (0 to 5$)$ according to definition of WMFT, we propose one modeling strategy. For the creation of this strategy (algorithm developed), we use the Mathworks MATLAB software which allows recovering in real time different types of documents (graphics, text, etc.). As a result, we are able to define an algorithm to analyze data and to evaluate the patient's physical activities according to the WMFT's scores.

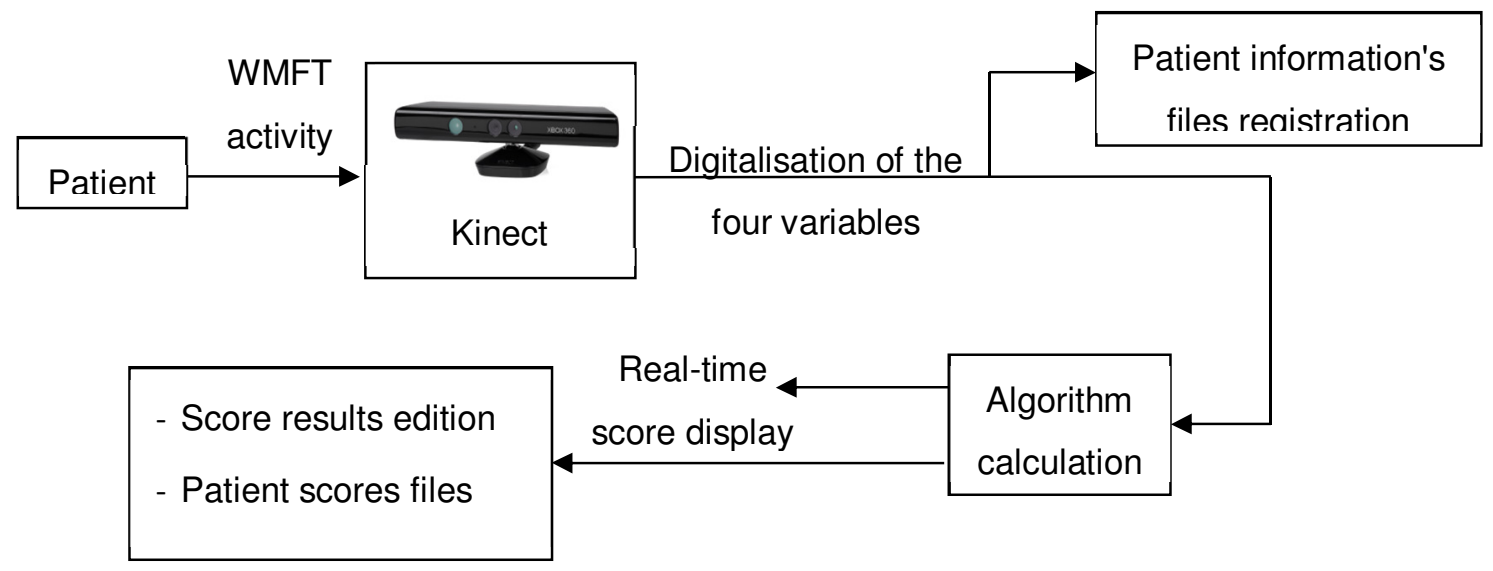

Figure 3. Diagram of the proposed measurement process in the WMFT research context.

To start our study, the fifth test of WMFT has been chosen [12]: Hand on the table, Functional capacity of the upper shoulder Member. According to measurement capabilities with the Kinect, this test is relatively simple (according to the medical specialists' opinion) and is required for this first stage of the research.

The description of the event is shown in Figures $4 \& 5$. The patient tries to put her hand on the table. The heel of the hand must stand beyond the drawn $2 \mathrm{~cm}$ from the edge of the table row. The palm of the hand does not need to be laid flat.

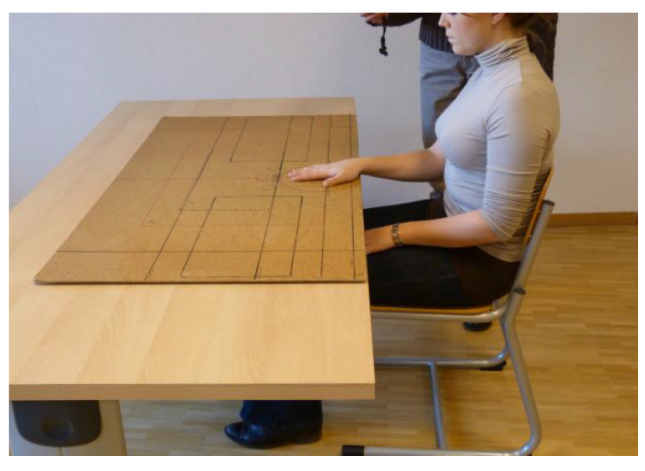

Figure 4: Start position.

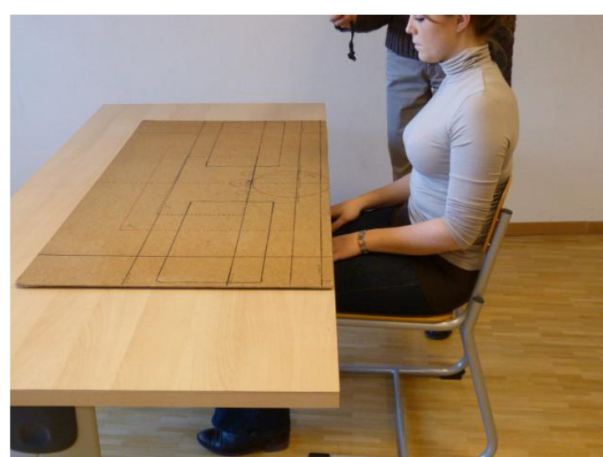

Figure 5: End position. 


\subsection{Scores Evaluation}

Patients who have to do the WMFT protocol must evaluate the two upper limb extremities. One upper limb is the affected one and they are not able to control it normally. The second part is the healthy part.

For the first discussion with the therapist in their points of views upon to figure 6: Kinect skeleton joints position. We understand that the joints of 'hand', 'wrist' and 'elbow' are the most challenged whereas the joints of 'shoulder', 'center of shoulder' are less challenged. The 'head' joint represented the least challenged.

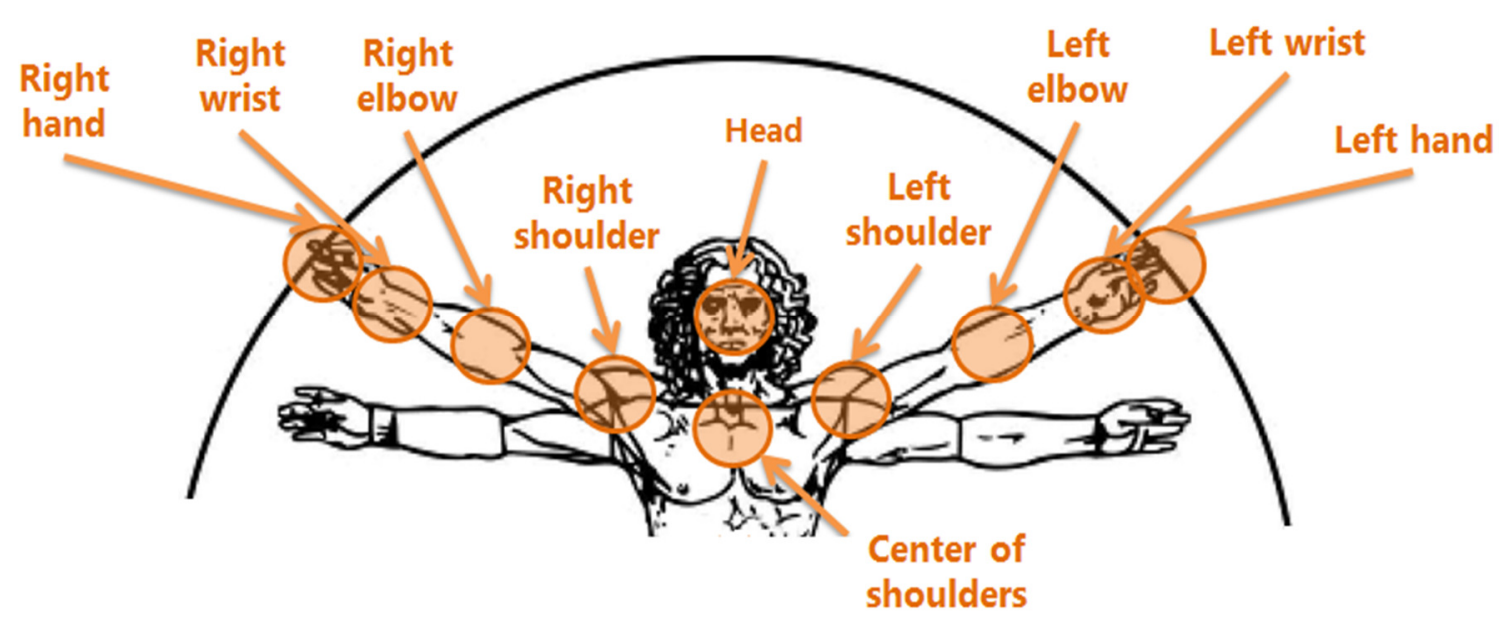

Figure 6: Kinect skeleton joints position.

According to definition of WMFT protocol for functional ability scale, the classifications for each scale have been done as below:

Score 0: As regards to the therapist's point of view, the description of the score 0 in the protocol WMFT and the outputs from Kinect, we have chosen the wrist joint for determining the score 0 .

Process of evolution: we defined the wrist joint in the position $\mathrm{Y}$. If the data from wrist joint in the position $\mathrm{Y}$ is zero, it means the patient was disabled to perform any movements, thus he or she took 0 score.

Score 1: In this step the concept from description of WMFT, it is the same as score 0 but the patient tried to perform the exercise ... but he or she was not able.

Process of evolution: we specified the threshold for the wrist joint in the position $\mathrm{Y}$. If the wrist joint in the position $\mathrm{Y}$ pass our threshold, the meaning that they do something and in this score it was important to try.

During discussions with Physiotherapists, definitions for scores 0 and 1 were decided according to the objectives. Scores 0 and 1 correspond to either no movement or wrist joint movement. It should be noted that sometimes patients cannot move their hand properly but little movements are detected in their shoulders. Therefore we added shoulder joint and elbow joint movements with value of these two scores.

Score 2: It will be given to someone who could raise his/her wrist up to the table.

Process of evolution: During the tests, both table and chair positions were fixed, so that all patients should sit in the same position, with same distance to the camera. We define the distance between the primary position of hand and the table in figure 7 . The objectives for score 2 is to see if the hand of the patient arrives to the table or not.

Score 2: It will be given to someone who could raise his/her wrist up to the table. 
Process of evolution: During the tests, both table and chair positions were fixed, so that all patients should sit in the same position, with same distance to the camera. We define the distance between the primary position of hand and the table in figure 7. The objectives for score 2 is to see if the hand of the patient arrives to the table or not

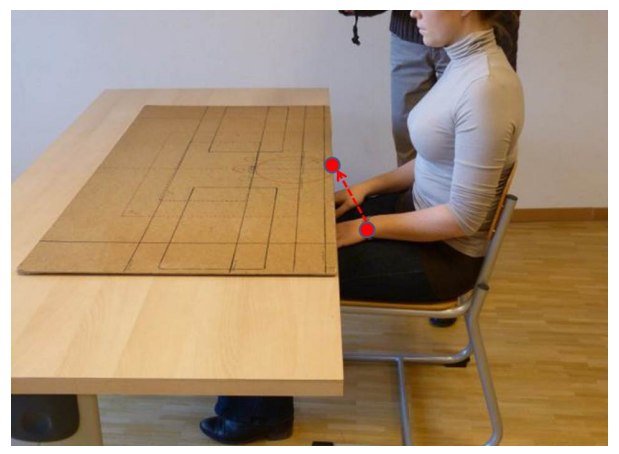

Figure 7: Movement of Wrist joint.

We calculated the difference between the maximum number and minimum number of wrist joint for each position and if the joint passes the borders, we can choose the score 2 for the patient

Score 3: The concept of this score is similar to score 2 but the patients showed better performance while their joints weren't come back.

Process of evolution: We realized the important challenge is the position $Z$, according to the knowledge of the therapist: The elbow and shoulder may be important if the patient does not have enough traction to flex his elbow to place his hand on the table. It will tend to spread the elbow from his body, to ascend the shoulders or to tilt the trunk to complete the task because they try to perform to the table with the help of his or her shoulders.

We chose shoulder right joint in position $Z$ for Left and right slide and calculate for each cell the difference in the same time in figure 8 and if they do not cross the border, the model takes a score 3 . The border should be determined by the therapist.

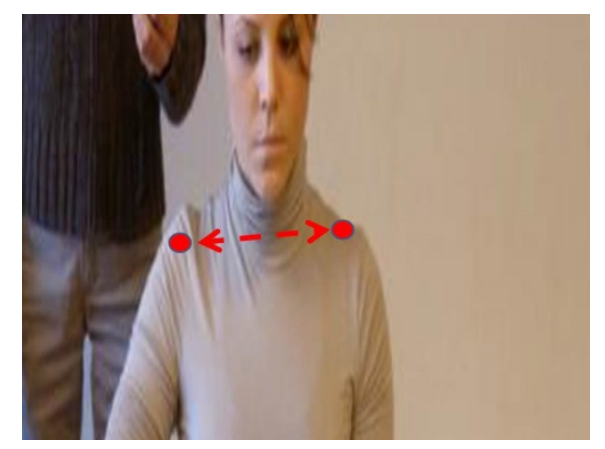

Figure 8: Distance between Shoulder joints.

To have comparison between scores 2 and 3 , we considered the score to be 2 if the wrist joint had a backward movement (almost equivalent to the patient having done the item two times) and to be 3 if movements of shoulders are detected in the position/direction $\mathrm{Y}$.

Score 4: The difference between score 4 and 5 according to the WMFT protocol and also therapist opinion is called" Fluidity ". Fluidity for the score $4 \& 5$ are qualitative parameters. At a first approximation, we are working on the speed parameter which is one of the points mentioned in the WMFT description.

We have the destination ( $\mathrm{X}, \mathrm{Y}$, and $\mathrm{Z}$ ) from Kinect output and also time. For the length we used Euclidean distance and know we have time also then with formula: Speed = Distance $/$ Time. 
To determine the score 4 and 5 , according to the description of WMFT protocol, we used the shoulder center joint in Z position to specify the score of 4 and speed for score of 5 .

If the patient gets score 3 , it mean is that he/ she could do this. We determined shoulder center joint in position Z, if the positions $Z$ change a lot during the time the meaning that, this patient has some problem and moves his/ her hand with help of the trunk.

\subsection{Modeling}

We have to imagine an algorithm that will transform the movement to descriptions suitable for physicians. In our case, the algorithm has to give a WMFT report for each patient. It allows giving the information of the patient's capabilities, in the required therapists' format.

We received 20 texts files, one from each joint. In the first step, we should arrange the text files. Afterwards, the goal is to analyze and then to evaluate the patient's physical activities according to the WMFT's standards. Since the scores of WMFT were divided by 6 steps, we have defined our process in 6 steps. The algorithm chooses zero for final score in first step. Then it will check the values according to the scenario which was defined for step 1 if the values satisfy the algorithm chose 1 for final score and go to next step for checking the other score, else the result of final score will be 0 and the algorithm will end. Such procedure continues and will be checked in each step until fifth step and if the procedures of checking the values that algorithm in the current step not satisfy the algorithm chose the last step for final score.

\subsection{Thresholds}

The above scores were given based on the following thresholds. These thresholds are defined based on WMFT's standards and are have been discussed with therapists.

We gave score 1 to patients who could move one of the wrist, elbow and shoulder joint by $2 \mathrm{~cm}$ from the first position. Score 2 was given to a patient who could rise his/her wrist up to the table. According to the score definition related to figure 7 , the threshold between hand and chair was defined equal to $(3$, $10,10 \mathrm{~cm}$ ). The score 3 was given to someone who is not able to twist her/his shoulders in position $Z$ for more than $6 \mathrm{~cm}$ and if there was a movement of the shoulder in the $Y$ position/direction y until $6 \mathrm{~cm}$. Score 4 was for patients who did not move her/his Shoulder Center joint in position Z more than $4 \mathrm{~cm}$. To increase accuracy in our calculation we have added this criterion to evaluate score 4 ; if the distance between the trunk and the elbow during the movement is less than $6 \mathrm{~cm}$, the patient is scored 4 . Finally, for score 5 we considered the speed the patient's action during the task. For this score the threshold was considered $6 \mathrm{~m} / \mathrm{s}$.

\section{Result}

We have tested our model with 10 persons in the G-SCOP laboratory. Participants are requested to do the tests in 5 configurations for the two sides of their body but eventually we asked them to perform the activity twice, once normally (first part) and then by assuming some physical challenges such as facing by the disabled people (second part). The results of these tests are available in table 1.

The second test was conducted without any limitations or prior definitions. In the data of the first part (except for person $\mathrm{F}$ and $\mathrm{J}$ that did not manage to do anything) all patients performed normally. According to the observation they did not confront any problem. The persons $\mathrm{F}$ and $\mathrm{J}$ did not change their position. Overall result of this part was illustrative of their activities but all of them could not get the score 5. After analysis of the data we understood the main reason was the speed of the action. Our definition for the speed in the score was too much $(6 \mathrm{~m} / \mathrm{s})$, the hence the average for 8 patients in healthy side was $4 \mathrm{~m} / \mathrm{s}$. Thus we put new measured average for next evaluations.

After doing the test on 10 patients with different conditions in the laboratory, observing and analyzing the scores, we came to the conclusion that the scores are quite accurate and sensitive to be defined for all of the scoring system; however these tests were done in the laboratory, in order for us to see how compatible the test is in real life and in the hospital environment, we took the test to the hospital. 
We asked the patients to perform the $5^{\text {th }}$ test of the protocol, once with the healthy side and the other time with the pathology side. Also we asked a physiotherapist to be present at the time of the test to give his/her scores too, to compare results.

For the first patient our model gave scores of 4 and 4 for healthy side and pathology side whereas the physiotherapist gave scores of 5 and 4 . With the same procedure we obtained the scores of 4 and 0 for the second patient in healthy side and pathology side contrasting the physiotherapist scores of 5 and 3.

The scores of the first patient is accurate from the point of the physiotherapist however as we see for the second patient there is a big difference between what the model and the physiotherapist gave for the scores, the reason for this was that the patients had a needle in his arm from the serum which made us unable to measure the performance and give a score.

Alongside with the evaluation of these score the model records three other data which are the time for which the patients take to do the activity, the changes of the elbow and evaluation of speed of the activity by the patients. According to the physiotherapist's recommendation it is beneficial for us to record those data as we can keep track of the patients progress.

\begin{tabular}{|c|c|c|c|c|c|c|c|c|c|c|}
\hline Person & A & B & C & D & E & F & G & H & I & J \\
\hline First Part & 4 & 4 & 4 & 4 & 3 & 1 & 4 & 4 & 4 & 0 \\
\hline Second Part & 3 & 1 & 3 & 3 & 2 & 1 & 3 & 3 & 4 & 0 \\
\hline
\end{tabular}

Table 1: The result of the model on 10 persons in G-SCOP laboratory.

\section{Conclusion}

In our study, we tried to understand WMFT protocol and their elements. Based on the studies, we used a system using Microsoft Kinect as an input tool to prepare a model that can be able to connect these two points. The obtained results of the research indicate that the proposed system is able to measure patients' physical capabilities required by WMFT protocol.

We chose Kinect based because previous researched showed Kinect to be accurate in medical applications. However this model needs few improvements such as Dynamic Time Warping algorithm and fuzzy logic so that we can compare two different parts of body alongside of the evaluations of the scores. The results of our work can be the base for the future research in the use of Kinect and implementation of their tools directly in the hospital of Grenoble. 


\section{References}

[1] L. Mündermann, S. Corazza, TP. Andriacchi, The evolution of methods for the capture of human movement leading to marker less motion capture for biomechanical applications, Journal of Neuroengineering and Rehabilitation 3 (2006).

[2] P. Horejsi, T. Gorner, O. Kurkin, P. Polasek, M. Januska, Using kinect technology equipment for ergonomics. 388/389. Analysis of human working postures designated for ergonomics, MM Science Journal (2013) 389.

[3] HP Oliveira, JS Cardoso, AT Magalhães, MJ Cardoso, "A 3D low-cost solution for the aesthetic evaluation of breast cancer conservative treatment," Computer Methods in Biomechanics and Biomedical Engineering: Imaging \& Visualization, 2(2), pp. 90-106, 2014

[4] Zolfagharnasab H, Cardoso J.S, Oliveira H. P. "3D Breast Parametric Model for Surgery Planning-a Technical Review," Proceeding of 22nd Portuguese Conference on Pattern Recognition, 1(1), pp. 85-86, 2013.

[5] RA. Clark, YH. Pua, K. Fortin, C. Ritchie, KE. Webster, L. Denehy, AL. Bryant, Validity of the Microsoft Kinect for assessment of postural control", Gait \& Posture 36 (3) (2012) 372-377.

[6] H. Gonzalez-Jorge, B. Riveiro, E. Vazquez-Fernandez, J. Martínez-Sánchez, P. Arias, Metrological evaluation of Microsoft Kinect and Asus Xtion sensors, Measurement, 46 (6) (2013)1800-1806.

[7] H. Jiang, X. Jie, Kinect-based Rehabilitation Training Assistant System Research and Implementation, International Conference on Software Engineering and Computer Science in ICSECS (2013) ISBN: 978-90786-77-82-6.

[8] T. Dutta, Evaluation of the Kinect_sensor for 3-D kinematic measurement in the workplace, Applied Ergonomics 4 (6) (2011) 645-649.

[9] C J. Su, JY. Huang, SF. Huang, Ensuring Home-based Rehabilitation Exercise by Using Kinect" and Fuzzified Dynamic Time Warping Algorith, Proceedings of the Asia Pacific Industrial Engineering \& Management Systems Conference (2012) 884-895.

[10]Wolf SL, Lecraw DE, Barton LA, Jann BB. Forced use of hemiplegic upper extremities to reverse the effect of learned nonuse among chronic stroke and head-injured patients, Experimental Neurology 104 (2) (1989) 125-32.

[11]Wolf SL, Catlin PA, Ellis M, Archer AL, Morgan B, Piacentino A, Assessing Wolf motor function test as outcome measure for research in patients after stroke, Stroke 32 (7) (2001) 16359.

[12]Rob Miles, Start Here! Learn the Kinect API, Publisher, the authorization of Microsoft Corporation by California 95472 (2012). 